\title{
Medición del desempeño en la cadena de abastecimiento del sector floricultor colombiano
}

\author{
Luis Martín Urrea Bello ${ }^{7}$ \\ Luz Gabina Garzón Cárdenas ${ }^{8}$ \\ Lucía Elena Pérez Suárez ${ }^{9}$
}

\begin{abstract}
Urrea, L., Garzón, L. \& Pérez, L. (2007). Medición del desempeño en la cadena de abastecimiento del sector floricultor colombiano. Revista Activos, 13, 15-46.
\end{abstract}

Recibido: 25 de marzo 2007 Aprobado: 29 de noviembre 2007

\section{Resumen}

Dada las condiciones de competencia mundial y la importancia del sector floricultor colombiano, el análisis de ventajas cómo la mano de obra barata, los bajos costos de transporte y almacenamiento, y de desventajas cómo el nivel de distribución e identificación de la flor, problemas tecnológicos que impiden el desarrollo de nuevas especies, y las restricciones internacionales en materia social, ambiental y técnica, hacen necesario el estudio de los diferentes eslabones de la cadena de abastecimiento de este importante sector. Lo anterior se materializa mediante el análisis de la planeación, las compras, la producción y la distribución, evidenciando aspectos importantes como el cálculo y gestión de costos, la utilización de mecanismos de medición del desempeño a partir de conceptos como los costos basados en actividades $\mathrm{ABC}$, y modelos de medición

\footnotetext{
${ }^{7}$ Contador Público de la Universidad Nacional de Colombia, especialista en Contabilidad Gerencial de la Pontificia Universidad Javeriana, docente en el área de Costos y Gestión de la Universidad Santo Tomás, lurreab@gmail.com.

${ }^{8}$ Contador Público de la Universidad Gran Colombia, especialista en Contabilidad Gerencial de la Pontificia Universidad Javeriana, director contable de importantes organizaciones del sector financiero y de valores como Bolsa Nacional Agropecuaria y Bolsa de Valores de Colombia BVC, luzgarzon2005@yahoo.com.

${ }^{9}$ Contador Público y especialista en Contabilidad Gerencial de la Pontificia Universidad Javeriana, docente en las universidades Central y Javeriana en el área de Gestión.
} 
de la gestión de la cadena de suministro como el modelo SCOR, el modelo gSCM, y la metodología de Balanced ScoreCard.

Palabras clave: Esquejes, Cadena de valor, Cadena de abastecimiento, Medición del desempeño, SCM gestión de la cadena de suministro, $\mathrm{ABC}$ costo basado en actividades, BSC Balanced Scorecard. JEL: G30, M11, M13, M41.

Urrea, L., Garzón, L. \& Pérez, L. (2007). Measurement of the performance in the chain of supplying of the sector colombian flower-grower. Activos Review, 13, 15-46.

\begin{abstract}
The conditions of world competition and the importance of the sector Colombian flowergrower, the advantages analysis how the cheap Labor, the low costs of transport and storage, and of disadvantages how the level of distribution and identification of the flower, technological problems that prevent the development of new species, and the international restrictions in social, environmental and technical matter, become necessary the study of the different links of the chain of supplying of this most important sector. The previous thing materializes by means of the analysis of the Planeación, the Buys, the Production, and the Distribution, demonstrating important aspects as the calculation and management of costs, the use of mechanisms of measurement of the performance from concepts as the costs based on activities $\mathrm{ABC}$, and models of measurement of the management of the chain of supply as the model SCOR, the model gSCM, and the Balanced ScoreCard methodology.

Key words: Cuttings, Chain of value, Chain of supplying, Measurement of the performance, SCM management of the chain of supply, ABC cost based on activities, BSC Balanced Scorecard. JEL: G30, M11, M13, M41.

Urrea, L., Garzón, L. \& Pérez, L. (2007). Mesure de la performance dans la chaîne d'aprovisionnement du secteur floricole colombien. Revue Activos, 13, 15-46.
\end{abstract}


ACTIVOS | REVISTA DE LA FACUltad de CONTAduría PÚBlica

\section{Résumé}

Face aux rudes conditions de concurrence mondiale et l'importance du secteur floricole colombien, l'analyse d'une part des avantages comme la main d'œuvre à bas prix, les coûts de transport et de stockage en baisse, et d'autre part les désavantages dans le niveau de la distribution et l'identification de la fleur sont les problèmes technologiques qui empêchent le développement de nouvelles espèces. Les restrictions internationales en matière sociale, environnementale et technique, font l'étude nécessaire dans différents maillons de la chaîne d'approvisionnement de ce secteur important. Ceux-ci prennent forme grâce à l'analyse de la planification, des achats, la production et la distribution, en mettant en évidence des aspects importants comme le calcul et la gestion des coûts. Ces formalités demandent aussi l'utilisation des mécanismes de mesure de la performance à partir des concepts comme les coûts basés dans les activités $\mathrm{ABC}$, et les modèles de mesure de gestion de la chaîne de fourniture comme le modèle SCOR, le modèle gSCM, et la méthodologie de Balanced scoredcard.

Mots clé: Les boutures, Chaîne de valeur, Chaîne d'approvisionnement, mesure de la performance, de SCM gestion de la chaîne d'approvisionnement, $\mathrm{ABC}$ coût basé sur des activités, BSC Balanced Scorecard. JEL: G30, M11, M13, M41. 


\section{Introducción}

El desarrollo de la industria nacional se enfrenta a verdaderos retos relacionados con acontecimientos que afectan la estructura económica y jurídica de la organización mundial. Dentro de un contexto de internacionalización económica, en el que las economías nacionales desaparecen para darle paso a las economías regionales y mundiales; la movilidad de los recursos y del capital financiero es la característica fundamental de las economías y en las cuales se configuran nuevos mecanismos propios de una sociedad del control, surgen las nuevas organizaciones, que deben estar en la capacidad de responder a los altos niveles tecnológicos de la industria mundial, a los bajos costos y, por ende, a bajos precios de bienes y servicios ofrecidos por la competencia y como es natural a las crecientes exigencias de los consumidores en términos de calidad.

No podemos desconocer que la economía colombiana actual se enfrenta a nuevas condiciones de competencia frente a la eventual firma del Tratado de Libre Comercio con los Estados Unidos, al igual que la posible presencia de futuros acuerdos que están pendientes de discusión con países tales como Chile, Perú, Ecuador, Venezuela, entre otros. Estos acuerdos de libre comercio crean grandes oportunidades para el país, pero no garantizan la prosperidad por sí mismos, porque se abren puertas a nuevos mercados para nuestros productos, pero así mismo para nuevos competidores, de allí la importancia de fortalecer los diferentes sectores económicos y prepararlos para competir en este nuevo entorno.

Dentro de los sectores más afectados con estas medidas se encuentra el sector agroindustrial. Las dificultades de nuestro país frente a los niveles de competencia que se avecinan y las pocas posibilidades de afrontar con éxito este reto son factores de riesgo, especialmente, teniendo en cuenta que uno de los sectores económicos con mayor desgravación arancelaria, mayores problemas de integración y tecnología, es el agroindustrial. Lo anterior exige al sector un cambio de estrategias, una preparación que permita subsistir, mantenerse y competir. "Todos los miembros del sector agrícola deberán examinar nuevas estrategias para ser exitosos" (Acero, 2003).

Se requiere entonces, un cambio en la forma de producir y de competir para este sector, que va desde la formulación de un plan de desarrollo, el análisis de 
integración, la reconfiguración de las cadenas productivas, la implementación de programas de educación y entrenamiento, al igual que la utilización de mejores prácticas, implementación de nuevas tecnologías y la utilización de modelos de gestión y medición del desempeño.

Como subsector perteneciente a la agroindustria colombiana, y por tanto afectado por estas preocupaciones, se encuentra la industria de las flores colombianas, cuyo análisis motiva el presente escrito, dada su importancia en la economía del país $\mathrm{y}$, adicionalmente, porque consideramos que este es un sector que tiene mucha proyección, si centra su atención en la búsqueda de eficiencia y optimización.

El sector floricultor colombiano, de acuerdo con el planteamiento de este escrito, debe buscar su sostenibilidad y competitividad a través de la gestión de su cadena de abastecimiento y en especial a partir de la medición de su desempeño.

Iniciemos entonces el estudio del sector que nos ocupa, con un análisis de su estado actual, ventajas y debilidades.

\section{Diagnóstico del sector floricultor colombiano}

Durante 40 años de desarrollo, el sector floricultor ha logrado el reconocimiento de las flores colombianas a nivel mundial por sus altos estándares de calidad, colorido, belleza, tamaño y variedad, posicionando a Colombia como el segundo país exportador después de Holanda, con una participación del 14\% en el mercado internacional.

Las principales zonas de producción en el ámbito nacional se encuentran ${ }^{10}$ en la Sabana de Bogotá (79\%), Antioquia (17\%) y otras zonas como el Valle, Eje Cafetero, Cauca (4\%).

El área cultivada en Colombia alcanza las 7.290 hectáreas, con Cundinamarca y Rionegro que suman 6.953 hectáreas.

${ }^{10}$ Cifras extraídas del reporte "Floricultura de exportación 2005". Últimas cifras de la floricultura colombiana. 
Otras cifras que respaldan la importancia de la industria de las flores en la economía colombiana son las siguientes:

Los mercados a los que exporta el sector son América del Norte, 83,1\%; Europa, $8,6 \%$; otros, $8,3 \%$.

Como podemos observar, el mercado con mayor importancia es el norteamericano, pero la fuerte dependencia de éste ha motivado la incursión en otros mercados no tradicionales, en la búsqueda de la sostenibilidad del sector.

A partir del estudio realizado por el Ministerio de Comercio, Industria y Turismo para este sector, la estructura en la producción de flores a nivel mundial señala que los principales productores de flor son Holanda con 7.378 hectáreas; los Estados Unidos con 20.181 hectáreas y Japón con 17.569 hectáreas; controlando aproximadamente el 50\% del valor de la producción mundial y más del $20 \%$ del área de producción. Es notable señalar que Colombia alcanza en hectáreas el 98,5\% del área ocupada por Holanda.

De igual forma, los grandes consumidores de flor del mundo son Europa occidental, Japón y Estados Unidos, con un total de 44.000 millones de dólares, aproximadamente. El mapa de las exportaciones señala que los principales abastecedores de flores a países orientales como Japón y Hong Kong son los países del Asia pacífico; los principales países abastecedores de Europa son Holanda y algunos países africanos, y los principales abastecedores de EE.UU son los países sudamericanos en especial Colombia.

Colombia exporta más de 50 tipos de flor, entre los cuales se destacan la rosa $(30,3 \%)$, el clavel $(13,5 \%)$, el clavel miniatura $(8 \%)$, el crisantemo y los pompones $(8,2 \%)$ y los bouquets $(26,4 \%)$. Para el 2001 la producción bruta de flores fue de USD 629.2 millones, con una participación en el PIB del 0,7\%, y con una generación de empleos directos de 75.000 personas para ese año. En la actualidad esta cifra supera los 300.000 empleos directos, ocupando a 4.500 profesionales en estas organizaciones. Vale mencionar también que este sector en la economía es el tercer generador de divisas después del café y el banano ${ }^{11}$.

\footnotetext{
${ }^{11}$ Cifras extraídas del reporte "Floricultura de exportación 2005". Últimas cifras de la floricultura colombiana. pp. 10-11.
} 
Revisemos entonces la evolución de las exportaciones de acuerdo con los datos de Asocolflores:

Tabla 1. Exportaciones de flores

\begin{tabular}{|c|c|c|c|c|}
\hline \multirow{2}{*}{ AÑ̃ } & \multicolumn{4}{|c|}{ EXPORTACIONES } \\
& \multicolumn{4}{|c|}{ Cifras en millones de dólares) } \\
\hline $\mathbf{2 0 0 6}$ & EE.UU & EUROPA & OTROS & TOTAL \\
\hline $\mathbf{2 0 0 5}$ & 781 & 82 & 77 & 940 \\
\hline $\mathbf{2 0 0 4}$ & 759 & 85 & 65 & 909 \\
\hline $\mathbf{2 0 0 3}$ & 597 & 62 & 45 & 704 \\
\hline $\mathbf{2 0 0 2}$ & 575 & 64 & 42 & 681 \\
\hline $\begin{array}{l}\text { Variación 2006- } \\
2002\end{array}$ & 568 & 62 & 41 & 671 \\
\hline
\end{tabular}

Fuente: Asociación Colombiana de Exportadores de Flores - ASOCOLFLORES.

Las exportaciones durante los últimos años han aumentado en un $40 \%$, con especial importancia en las exportaciones a otros mercados representados por Asia y Europa Oriental. La evolución de las exportaciones en este período se observa en el siguiente gráfico.

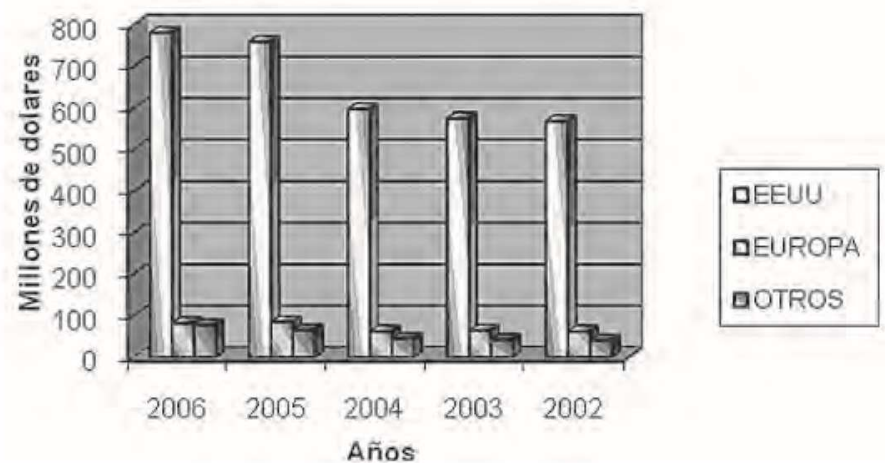

Figura 1. Evolución de las exportaciones

Fuente: Asociación Colombiana de Exportadores de Flores - ASOCOLFLORES. 
Colombia cuenta con fuertes ventajas para la producción de flores, con respecto a su principal competidor, Holanda. Ventajas que debe saber aprovechar, como:

- La inexistencia de estaciones

- La abundancia en recursos acuíferos

- La gran variedad de especies

- La luminosidad adecuada

- El clima, la topografía plana, tierras fértiles y pisos térmicos, temperatura óptima para producción en invernadero

- Mano de obra relativamente barata y bajos costos de transporte y almacenamiento por cercanía a los puertos de embarque (aeropuerto El Dorado y el de Rionegro).

Resaltemos algunas otras características de la producción que definen este sector de la economía colombiana, de acuerdo con el estudio: Comportamiento reciente del sector floricultor colombiano. Tenjo, F, Montes, E \& Martínez, J. (20002005).

- La producción de flores en Colombia es realizada principalmente por Pymes que exportan de manera atomizada. El 90\% del valor total exportado en 2004 (US\$704 millones) fue transado por 228 empresas cada una de las cuales exportó, en promedio, más de US\$3 millones.

- La producción de flores se destina básicamente al mercado externo y las ventas tienen un comportamiento estacional.

- Las importaciones realizadas por el sector floricultor son principalmente agroquímicos y esquejes.

- Los esquejes importados en su mayoría desde Holanda incorporan el factor de investigación y desarrollo del sector, incluyendo nuevas tendencias de mercado y de producción.

- El ciclo de producción de las flores en Colombia depende de la variedad cultivada: las rosas se cosechan cada 90 días y cada rosal tiene una vida útil hasta de 15 años. En el caso de los claveles, la cosecha se efectúa con una periodicidad semestral y la planta dura dos años en producción.

Pero si esta industria ha ganado terreno dentro de las exportaciones mundiales, incrementando el nivel de flores despachadas (volumen embarcado), ocupando

ISSN: 0124-5805- No. 13 - 2007 - pp. 15-46 | 23 
un importante lugar en la generación de divisas nacionales y convirtiéndose en un importante generador de empleos directos e indirectos, también es cierto que, en años recientes ésta se encuentra atravesando una crisis financiera, representada por disminuciones en las utilidades, esencialmente causadas por problemas en la variabilidad del dólar. Los procesos de revaluación del peso frente al dólar, la dependencia de los ingresos con relación a los precios internacionales de las flores y la dependencia de los gastos a la variación de precios internos, son las causas principales de la disminución de las utilidades.

Este comportamiento lo podemos observar en la siguiente Figura.

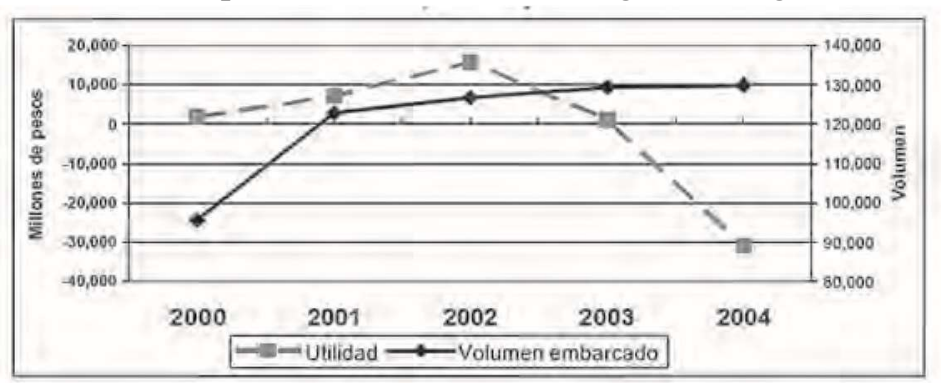

Figura 2. Utilidades antes de impuestos y volumen embarcado

Fuente: Fernando Tenjo G., Enrique Montes U., Jorge Martínez (2006), 12.

Sin embargo, existen otros problemas que son importantes de entender a la hora de pensar en las fuertes oleadas de competencia que se avecinan para Colombia y para las industrias que conforman su economía. Los problemas que enfrentan estas organizaciones en la actualidad son los siguientes:

\section{Crecimiento en la competencia externa}

Los productores y exportadores de flores a nivel mundial son cada vez mayores ya que se están incorporando países como Kenia, Ecuador y Zimbabwe, que gracias al bajo costo de la mano de obra y de la tierra, y dadas sus características climáticas, han incrementado enormemente sus exportaciones y su participación en el mercado mundial. 


\section{Distribución y conservación de la flor}

Este problema hace referencia al manejo de la distribución de las flores, en especial, lo relacionado con la conservación de este producto. Se presentan problemas en el transporte de las flores, que requieren de sistemas de conservación en fríos y que dada la estructura de esta cadena, no se cuenta con las tecnologías para la conservación adecuada de los productos y el mantenimiento de los estándares de calidad. Adicionalmente, la cadena de distribución es muy extensa, dependiendo de mayoristas y minoristas, que por lo general están fuera del alcance de los productores colombianos, volviendo éste un proceso desconectado entre la demanda y la oferta, con pérdida de control sobre la demanda y con períodos de entrega muy largos.

Se señala que "no existen sistemas integrados: transporte, almacenaje y distribución, son actividades diferentes. No hay un verdadero proceso de distribución; sólo es una sumatoria de tareas. Puede pasar más de ocho días desde que sale del cultivo hasta que llega al punto de venta, demasiados eslabones en la cadena que hace perder tiempo precioso" (Carranza, 2005).

\section{Problemas tecnológicos}

Dentro de los problemas técnicos y tecnológicos tenemos la baja investigación y desarrollo, que impiden la generación de productos nuevos y de técnicas de producción que permitan reducir los costos o mejorar los niveles de calidad de la flor o las variedades de las mismas. Los esquejes, reflejo de los esfuerzos en investigación y desarrollo, son producidos principalmente por los países competidores, Holanda e Israel, lo que lleva a los empresarios colombianos a competir con un producto no diferenciado, lo cual es importante en la toma de acciones para mantener los mercados actuales y la penetración de nuevos.

\section{Restricciones internacionales}

Se presentan restricciones de carácter ambiental, social y técnico. Por ejemplo, Japón y el mercado europeo exigen a los productores colombianos procesos industriales y comerciales que garanticen mejores condiciones sociales en contratación laboral y en impactos ambientales. De igual forma, se presentan 
ACTIVOS | REVISTA de LA FACULTAD DE CONTADURía Pública

controles de carácter fitosanitario y controles para prevenir el tráfico de drogas. Estas restricciones incrementan los costos de generación y distribución de la flor.

\section{Otras debilidades}

Como resultado del levantamiento de información realizada mediante visitas a empresas del sector, se evidenció la existencia de otras debilidades, ellas son:

1. Se carece de las herramientas apropiadas que permitan gestionar el negocio, de manera adecuada, desarrollando así actividades de forma desordenada y sin un plan de trabajo; en ocasiones parece que actúan de manera reactiva con los clientes, tratando de satisfacer la demanda actual, sin considerar la posibilidad de ampliar el horizonte, mediante el crecimiento del mercado

2. No disponen de sistemas de información que les permitan tener un adecuado conocimiento de los clientes actuales y potenciales

3. No se evidencia ninguna ambición, que les impulse a la conquista de mercados, a la optimización de recursos y al incremento de valor

4. No miden la gestión que están desarrollando, ya que no cuentan con indicadores, desconociendo los avances y las técnicas que en estos términos existen.

Como podemos observar, los anteriores problemas nos invitan a pensar en cómo fortalecer las estructuras operativas y cómo lograr estrategias que permitan mejorar la competitividad de la industria de las flores colombianas. Nuestro planteamiento es que los niveles óptimos de competitividad para el Sector se pueden lograr con una mejor Administración de la Cadena de Abastecimiento o Supply Chain Management-SCM-, (traducción en inglés). Pero esto plantea no sólo administrar sino evaluar su desempeño, es decir, medir y comparar, realizar un proceso de benchmarking que nos ayude a comprender por qué Holanda con tan poco territorio cultivable, es el mayor exportador de flores en el mundo y tiene una participación en el mercado superior al 50\%, porcentaje que triplica la nuestra, que apenas logra un $14 \%$.

Para adentrarnos en nuestro estudio es necesario que describamos inicialmente la cadena de valor del sector floricultor colombiano. 


\section{Cadena de valor}

La cadena de valor de este sector comienza con el diseño y desarrollo de las distintas variedades de flores, para así realizarse un análisis de los gustos de los consumidores, tendencias del mercado y resistencia de plagas, es desde ésta que se refleja la investigación y desarrollo del sector. La siguiente actividad "Adquisición de esquejes", se describe como el proceso de compra que realiza cada organización (cultivo) desde la escogencia de la variedad hasta la negociación de patentes y respectivo acuerdo de regalías. Paso seguido vienen las actividades que comprenden el proceso productivo desde la propagación de material vegetal hasta el empaque de las flores, luego del empaque es importante la conservación de las flores con la actividad de "Almacenamiento en cuarto frío". Finalmente, se presentan las actividades de comercialización, despacho y distribución.

Visualicemos entonces una descripción gráfica de todas las actividades que comprenden la cadena de valor para el sector de acuerdo con nuestro análisis:

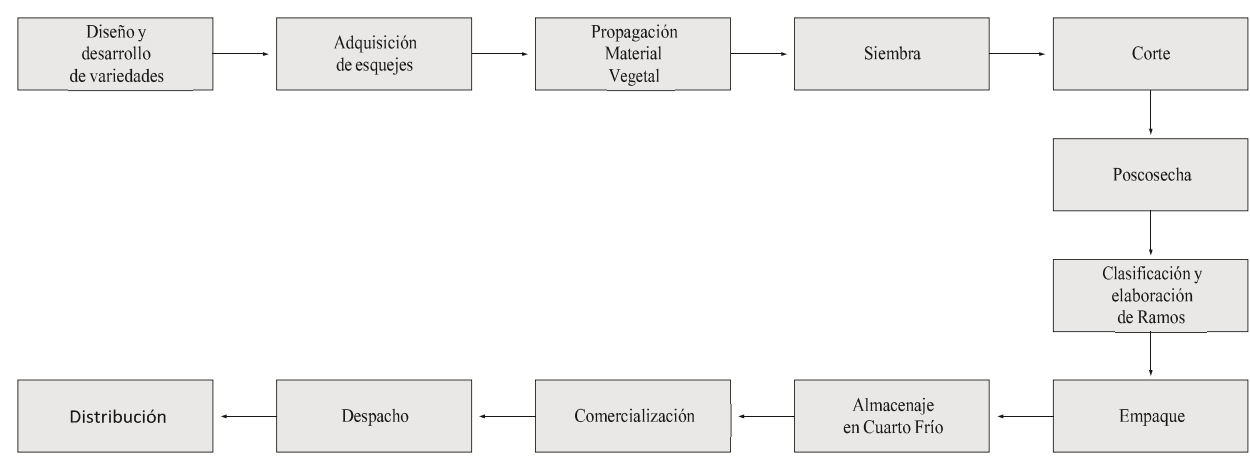

Figura 3. Cadena de valor del sector floricultor colombiano Fuente: elaboración propia.

Cuando analizamos la cadena de valor pensamos en el margen de la cadena, es decir, la diferencia entre los costos de las actividades y el costo al que le ha llegado al consumidor final. En la cadena de valor de las flores donde se generan mayores márgenes es en la actividad de distribución al consumidor final. Siendo un producto de exportación cuando las flores colombianas llegan a su destino en el exterior, intervienen varios actores antes de llegar al consumidor final, como analizamos en la cadena de abastecimiento siguiente. 
ACTIVOS | REVISTA de LA FACULTAD DE CONTADURía Pública

\section{Ahora bien, ¿qué entendemos como administración de la Cadena de Abastecimiento?}

Debemos entender en qué consiste la Cadena de Abastecimiento. Ésta se relaciona con "... todos los recursos interconectados y las actividades necesarias, para crear y entregar productos y servicios a los clientes. En el sentido más estricto la Cadena de Abastecimiento se extiende desde el punto donde se extraen los recursos naturales de la tierra hasta el punto en donde se regresan a la tierra" (Bill Hakanson, 2003). Se debe entonces planear, implantar y controlar el flujo y almacenamiento de productos e información de la cadena de forma tal que seamos costo - efectivos, en procura de maximizar el resultado global de la cadena y, por ende, ser más competitivos.

Cuando se habla de CADENA DE ABASTECIMIENTO, de ante mano, se supone que se desea sincronizar el flujo de productos y dinero desde proveedores hasta clientes, sin buscar el beneficio particular de alguno de los miembros. Ello está precisamente alineado con la necesidad de encontrar un óptimo global del sistema y no uno parcial de alguna compañía o función (Mariño, 2003).

De acuerdo con esto, y en procura de encaminar nuestra propuesta, consideramos que debemos dejar de pensar como entes individuales, para poder integrar a todos los participantes de la cadena de forma tal que nos olvidemos del concepto de regiones y pensemos más como país.

Como explicábamos anteriormente, con relación a la Cadena de Abastecimiento, aplicada a las flores colombianas, comprende desde la compra de insumos agrícolas y esquejes, hasta la entrega al cliente final, o, extendiéndonos un poco hacia el concepto ambiental, comprendería hasta la disposición por el consumidor final de las flores muertas y su empaque. 


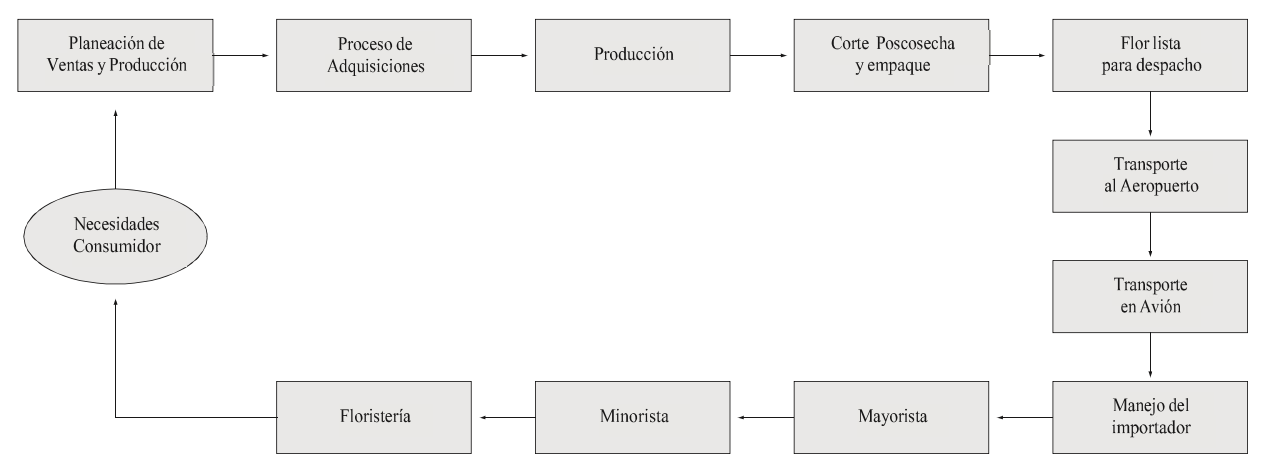

Figura 4. Cadena de suministro Fuente: elaboración propia.

Para un conocimiento acertado de la SCM, debemos comprender de que está compuesta la cadena de suministro.

\section{Componentes de la Cadena de Abastecimiento}

\section{Planeación}

En relación con la cadena de suministros, permite determinar las mejores formas de comprar, fabricar y distribuir los bienes y servicios, señalando cuanto se debe producir, qué recursos demanda, en qué momentos se va a producir y bajo qué parámetros se va a distribuir. Este proceso permite equilibrar la demanda y el suministro de tal forma que se alcance los objetivos planteados en el negocio.

En el sector floricultor la planeación debe tener en cuenta aspectos como:

- Aspectos climáticos.

- Las necesidades del cliente que señalan que el consumo depende de las fiestas programadas durante el año.

- Las condiciones particulares definidas por el cliente. Vale la pena mencionar los requisitos que se exigen en Japón, donde las flores se deben entregar en botón.

- Las cantidades de flores demandadas por cada país y dadas las condiciones de la competencia. 
ACTIVOS | REVista de la Facultad de CONTAduría PÚBlica

\section{Compras}

Esta actividad comprende la selección de las variedades, estableciendo los colores que se van a sembrar. Los proveedores de material vegetal, los precios, las variedades precoces, las variedades resistentes, la compra de plantas madre material vegetal para enraizar y producir esquejes, los fertilizantes, fungicidas, plaguicidas, material para la fabricación y conservación de invernaderos y el establecimiento de las fechas de entrega de los insumos. En este componente se requiere un conocimiento acertado de los proveedores o una buena selección del proveedor de manera que esto asegure calidad, oportunidad y eficiencia al proveer los insumos para la siguiente etapa.

\section{Producción}

El proceso productivo del sector de las flores se realiza a partir de la estrategia de fabricación contra pedido. En la cadena de suministro se determinaron el conjunto de actividades que configuran los procesos productivos. De acuerdo con lo anterior observamos los siguientes eslabones de la cadena productiva:

Propagación de material vegetal. En este proceso se realiza la adecuación del área de propagación, encontrando el área de plantas madre, que incluye: la preparación de las camas para sembrar las plantas madre; la siembra y labores de riego, fumigación y fertilización; la cosecha y almacenamiento de esquejes sin raíz; y el área bancos de enraizamiento, que incluye: la preparación de los bancos de enraizamiento; la siembra de esquejes sin raíz; las labores de riego; la fumigación y fertilización; la cosecha y almacenamiento de esquejes con raíz.

Presiembra - Producción en hidropónico. Consiste en actividades como: hechura de camas, riego para humedecer sustrato e instalación de equipo de riego por goteo.

Siembra. Las actividades que caracterizan este proceso son la siembra, que incluye la colonización del material vegetal -esquejes con raíz- en los surcos de las camas; el riego para refresque que se realiza con micro aspersores, aspersores, al igual que el fertirriego considerado como riego más fertilización (se realiza en el período vegetativo y reproductivo); proceso de fumigación, se aplica semanalmente fungicidas y plaguicidas contra plagas, enfermedades y bacterias. 
Labores culturales. Consisten en el desarrollo de actividades tales como despunte, encanaste, desbotone, encauche, desencauche y proceso de fumigación.

Corte de la flor y transporte a poscosecha. En el desarrollo de este proceso se realizan las siguientes actividades: corte a partir de los requerimientos del cliente, como: Europa apretado, Europa semicopa, copa (flor abierta), pétalo recto; la acomodación en cajas para envío a poscosecha; envío a poscosecha utilizando sistemas como el envío en seco y el envío húmedo a través de cable vía o vehicular.

Poscosecha. En este lugar es donde se realizan la recepción de la flor y su alistamiento para la actividad de clasificación y elaboración de ramos. Para el desarrollo de estas actividades se deben tener en cuenta las siguientes condiciones: tamaño de las salas, donde se debe observar el manejo adecuado de espacios para el desarrollo óptimo de las operaciones; la temperatura, aireación y humedad, en busca de evitar las altas temperaturas que causa la aglomeración de etileno y la deshidratación de la flor; la iluminación que garantice un buen desempeño de los operarios.

Dentro de la infraestructura a utilizar se debe contar con: carros de corte, que eviten maltrato de las flores; soportes para cajas o lonas; mesas de clasificación; recipientes para el tratamiento; cuartos fríos; zonas de carga; máquinas, equipos y controladores para hacer mucho más eficiente la operación. Por ejemplo, la utilización de máquinas clasificadoras, bandas transportadoras y pistolas neumáticas.

En esta etapa se debe, adicionalmente, realizar actividades de control de calidad y recepción de la flor del cultivo, en la cual se verifica la calidad, cantidad y procedencia, y establece la información necesaria sobre la flor que ingresa a la sala.

Clasificación y elaboración de ramos. En este caso se desarrollan las siguientes actividades: registro de las cantidades de flor por áreas, variedades, distribución a clasificadoras; proceso de clasificación, teniendo en cuenta calidades de exportación o distribución nacional, variedades, colores, y rendimientos por clasificadores; elaboración de ramos; inspección de ramos; tratamiento de los 
ACTIVOS | REVISTA de LA FACULTAD DE CONTADURía Pública

ramos teniendo en cuenta calidad del agua, la composición química del tratamiento, dosis y duración del tratamiento y la asepsia de los recipientes.

Empaque. Este proceso se realiza con base en los pedidos de los clientes y se tienen en cuenta las cantidades por ramos, las cantidades por caja, los tipos de cajas, los grados de apertura, la marcación de cajas con datos en etiqueta de código de barras. Los tipos de empaque son por flores enteras, combos (mezcla de flores en cajas) y bouquets (mezclas de flores por ramos).

Almacenaje en cuarto frío. En este proceso se realiza las siguientes actividades: entrada de flor a cuarto de pre enfriamiento; entrada de flor a cuartos fríos; ubicación en estanterías para la salida de la flor por despachos o bajas (por mercadeo, por calidad, por envejecimiento) (ver Figura 3).

\section{Distribución}

En este proceso se realizan las siguientes actividades en las operaciones de exportación: Trámites de exportación, registro de exportación, colocación de etiquetas para marcación de cajas, consecución de cupos en compañías aéreas y la elaboración de documentos.

La cadena de distribución no termina con el cargue de las flores, se debe tener adicionalmente el transporte y la entrega a los diferentes clientes.

Para el caso de los EE.UU, la distribución se realiza teniendo en cuenta agentes como los mayoristas y los minoristas, pasando por las floristerías hasta llegar al consumidor final. En este caso, todo el proceso de transporte está a cargo de terceros (empresas comerciales) que no cuentan con los esquemas de fríos necesarios para la conservación óptima de las flores.

\section{Los costos en la cadena de abastecimiento}

Dentro de nuestro estudio consideramos de gran importancia determinar el estado actual de la cadena a través del cálculo del costo de las actividades que se desarrollan en ella, es decir, la empresa debe conocer cuánto le cuesta realizar una compra, producir las unidades para vender, despachar un pedido, entre otros. Dentro de las herramientas que permiten determinar los costos, encontramos el 
Costeo ABC, como una de las más acertadas para este propósito, por esta razón explicaremos de manera breve en qué consiste.

\section{Costos ABC y la SCM}

La aplicación del costeo $\mathrm{ABC}$ en la administración de la cadena de abastecimiento permite establecer el costo de desarrollar cada proceso $\mathrm{y}$, con ello, cada una de las actividades de la cadena. Lo anterior nos permite determinar cuánto cuesta planear, comprar, producir, entregar, devolver, para cada participante de la cadena y para toda la cadena en general.

Para implementar el sistema de costeo ABC necesitamos entonces (Insitute of management Accountants, 1992):

- Definir las actividades y frecuencias para cada proceso o función logística

- Una vez entendamos éstas, es necesario determinar ciclos de tiempo y costos para las actividades

- Identificar los cost drivers o inductores de costo para las actividades logísticas, podemos guiarnos por los siguientes ejemplos:

Número de referencias (stock keeping units) de materiales

Niveles de inventario

Número de bodegas

Transporte

Almacenaje y manejo

Número de despachos

Número de devoluciones

Es importante entonces entender en la cadena de abastecimiento por qué se generan los costos, cómo y cada cuánto.

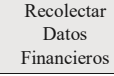


ACTIVOS | REVISTA DE LA FACUltad DE CONTADURía PÚBlica

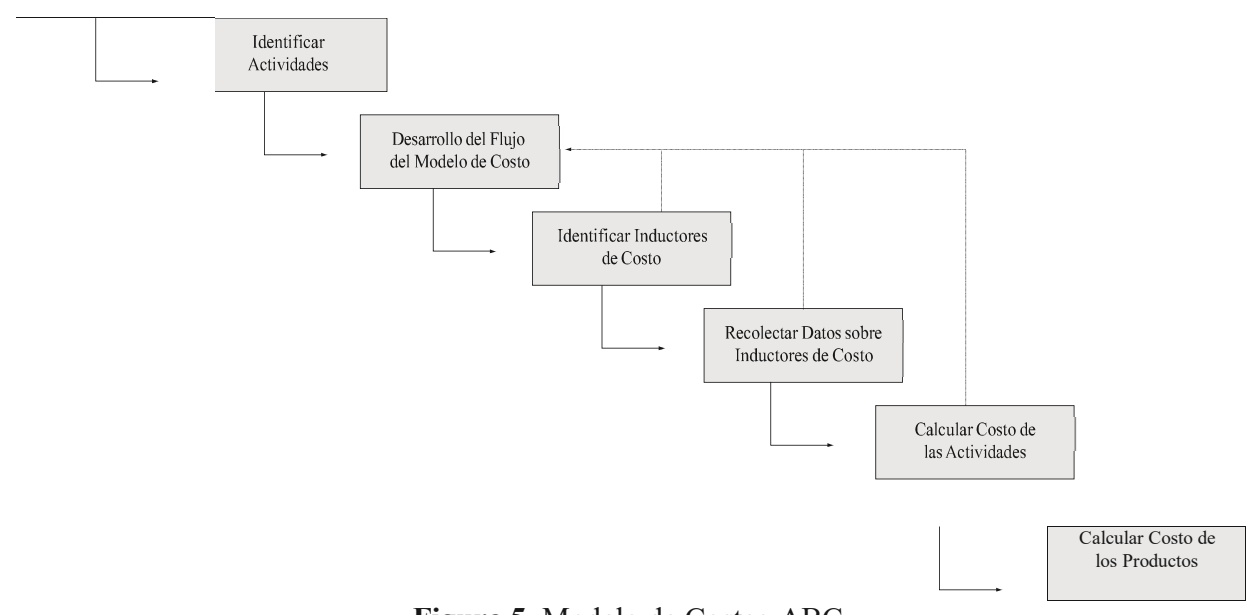

Figura 5. Modelo de Costeo ABC

Fuente: elaboración propia.

\section{¿Cómo medir el desempeño en la Cadena de Abastecimiento?}

Dirigidos a cumplir con los objetivos de esta investigación, consideramos nuestro mayor valor agregado la propuesta de medición del desempeño de la cadena de abastecimiento que planteamos a continuación.

La propuesta consiste en la integración de tres alternativas, así: el modelo gSCM, el modelo SCOR y finalmente el Balanced Scorecard. Esta integración permitirá obtener los mejores beneficios ya que cada uno de éstos direcciona a la organización hacia estrategias importantes dentro de la cadena. A continuación, se hace una breve explicación de cada una de las alternativas.

\section{Modelo gSCM}

Dentro de las metodologías para implementar la administración de las cadenas de suministro, encontramos una de éstas que ha sido desarrollada en Colombia y probada a nivel mundial, con resultados altamente satisfactorios, ya que permite identificar las grandes barreras que se presentan cuando las empresas inician la implementación práctica de SCM.

La metodología presenta en esencia tres componentes, en el que cada uno de éstos define con claridad un entorno competitivo para la organización, en la que se identifican una serie de puntos positivos o negativos que posteriormente serán 
analizados para configurar la cadena de suministro, logrando un alto nivel de eficiencia.

En cada una de estas etapas se deben evaluar aspectos, tales como: el macroeconómico, el tecnológico, el cultural, la infraestructura, la normatividad, los competidores, los inversionistas, el posicionamiento de la empresa, entre otros.

De esta manera, se obtiene un resultado en el que se evidencia con claridad el perfil en el que se mueve la compañía en la actualidad para implementar, tanto la administración eficiente en su cadena de suministro, así como el plan de mejoramiento que se deberá aplicar en el corto, mediano y largo plazo. Con ello, se busca medir los resultados parciales para ir evaluando su aplicación y, por ende, alcanzar el logro de los objetivos propuestos en la organización.

\section{El modelo SCOR}

El modelo SCOR denominado como el modelo operacional para la cadena de suministro, es considerado un estándar que está enfocado en la administración de la cadena de suministro, sirviendo como instrumento de diagnóstico, que integra principios de reingeniería, benchmarking y elementos de medición del desempeño en los procesos de la cadena. Este esquema permite a las organizaciones diseñar e implementar planes de mejoramiento en el corto, mediano y largo plazo, al igual que la implementación de mejores prácticas universales de SCM, integrando el recurso humano, los procesos, las mediciones y la tecnología. Este modelo integra las actividades que van desde el proveedor del proveedor, y termina en el cliente del cliente, integrando así los procesos de planeación y de ejecución a lo largo de la cadena.

Los procesos integrados son:

- Planeación

- Compras

- Producción

- Entregas

- Devoluciones 
ACTIVOS | REVISTA de LA FACULTAD DE CONTADURía Pública

En el primer proceso se realiza la planeación de la cadena, de las compras, de la manufactura y la de entregas. Para los procesos de compras, manufactura y entregas se puede organizar según la estrategia industrial, clasificándose en:

- Adquirir para stock, adquirir para pedido, adquirir para diseño •

Fabricar para stock, fabricar para pedido, fabricar para diseño

- Entregar para stock, entregar pedido y entregar para diseño.

Este modelo trabaja cuatro flujos a saber: los flujos de materiales, información, dinero y decisiones; y se desarrolla en tres etapas: integración, sincronización y optimización.

En cuanto a las decisiones estratégicas, se toman en las siguientes áreas:

- Desempeño en entregas

- Desempeño en el cumplimiento de pedidos

- La capacidad de reponer

- Ciclo en el cumplimiento del pedido

- Cumplimiento acorde del pedido

- Tiempo de respuesta de la cadena

- Flexibilidad de la producción

- Costo total de la cadena de suministro (utilizando ABC)

- Ciclo en el manejo de efectivo • Rotación de inventarios • Retorno de activos.

En este modelo se plantea la implementación de mejores prácticas en el desarrollo de las diferentes actividades a lo largo de la cadena. Las mejores prácticas hacen referencia por ejemplo: a las formas de gestionar las compras de materiales; la forma de realizar la planeación de las compras o de la producción, utilizando esquemas de ERP y MRP, que garantice la optimización de los recursos; la forma de integrar los diferentes agentes industriales (empleados, proveedores, administradores y clientes) en procura de la obtención de los objetivos, la utilización de sistemas de información, la generación de indicadores de desempeño a lo largo de la cadena, la implementación de estrategias como el benchmarking, JIT, tercerización, entre otros. 
Así mismo, este modelo involucra indicadores que miden el desempeño financiero y operativo en la cadena. Entre los financieros tenemos, el crecimiento en venta, el cumplimiento de presupuestos, el manejo de costos, las variaciones de los costos reales con relación a los costos estándar, el cumplimiento del presupuesto de costos y gastos; entre los operativos tenemos, el porcentaje de cumplimiento de pedidos, los pedidos entregados a tiempo y en las condiciones especificadas, el número de errores en las actividades productivas, los niveles de rotación de inventarios, el nivel de utilización de la capacidad productiva, productividad de empleados, entre otros.

De acuerdo con lo anterior, el modelo SCOR completo se presenta así:

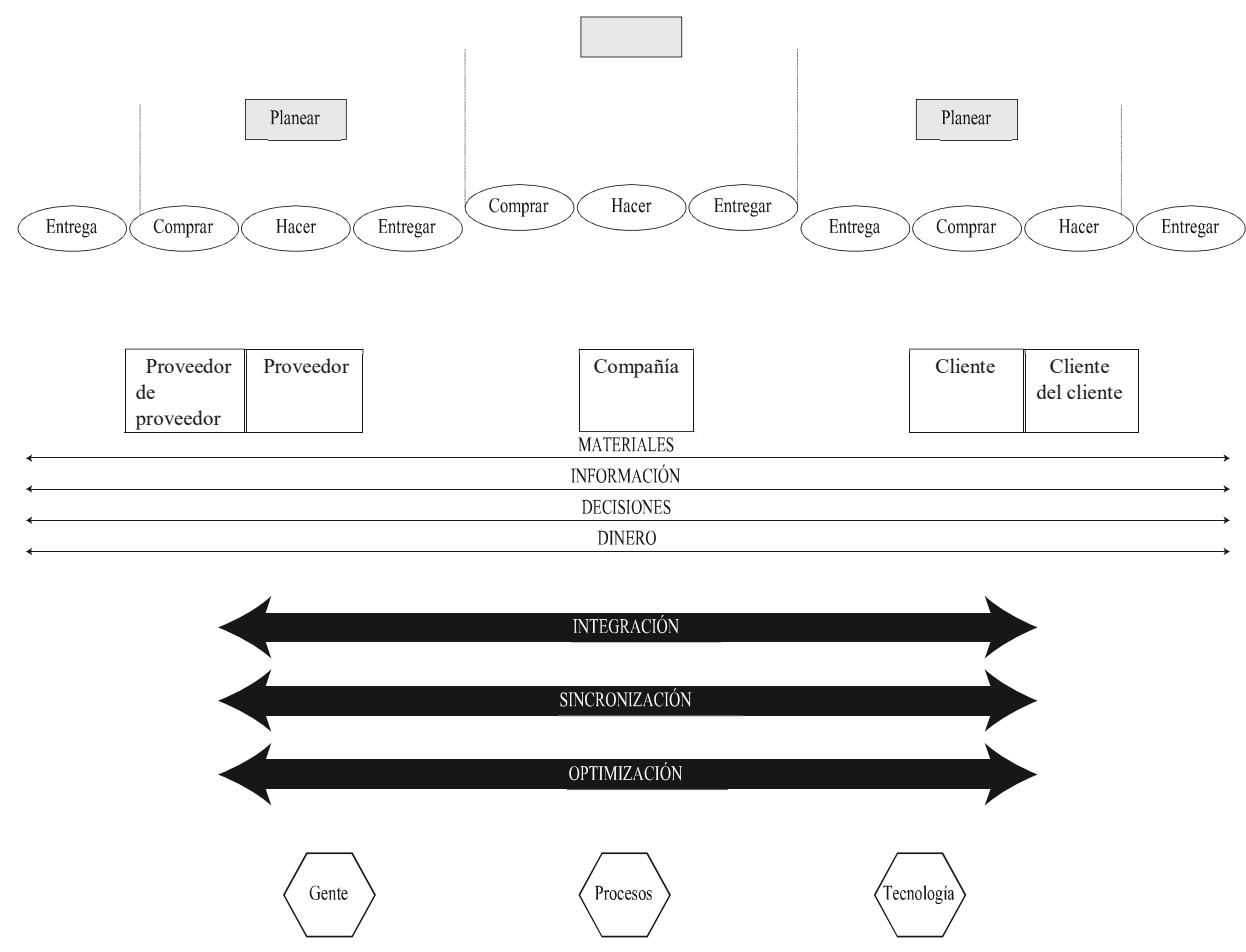

Figura 6. El Balanced Scorecard y el SCM Fuente: elaboración propia.

\section{El Balanced Scorecard}

El Balanced Scorecard (BSC) es un modelo de gestión que nos permite alinear estratégicamente una organización, pretendiendo traducir su estrategia en 
acciones. ¿Y esto cómo se logra? Entendiendo que la estrategia de la organización abarca más que aspectos puramente financieros, por tanto, se señala que los objetivos estratégicos pueden organizarse en cuatro perspectivas principales, adaptadas o modificadas de acuerdo con la organización:

- Perspectiva de aprendizaje y crecimiento. Comprende aspectos referentes a la mejora del capital humano y del capital estructural de la organización. Se trata de dejar claro qué bases estructurales en cuanto a gente y tecnología necesita la organización para soportar su operación.

- Perspectiva de procesos internos. Mediante el análisis de su cadena de valor la organización define los objetivos que son importantes dentro de su operación para satisfacer las necesidades de inversionistas y clientes.

- Perspectiva de cliente. Por medio de ésta se resaltan objetivos necesarios para cumplir con la promesa de valor al cliente.

- Perspectiva financiera. Se trata de señalar los objetivos principales para cumplir a los inversionistas o accionistas con lo que ellos esperan de la organización, mayor retorno de la inversión, mayores utilidades, entre otros.

Para cada perspectiva deben señalarse objetivos estratégicos principales de la organización, pero además debe tenerse en cuenta que por cada objetivo trazado debe existir un indicador de gestión que mida su desempeño y un plan de acción para mejorar o mantener dicho desempeño.

Para comunicar la estrategia, el BSC se vale de los mapas estratégicos que demuestran gráficamente cómo entiende la organización la interconexión entre sus objetivos estratégicos, es decir, qué objetivo es necesario para lograr otro, relación de causa y efecto, mostrando así cómo se alinean las perspectivas.

La estrategia de SCM debe alinearse con la estrategia de la organización, deben hablar el mismo idioma y a través del BSC podemos realizar esto, mostrando los objetivos trazados para cumplir con la primera, que comprende aspectos tanto financieros como no financieros, e interrelacionarlos con los objetivos que pretenden cumplir la otra.

\section{¿Cómo medimos el desempeño en la cadena de abastecimiento del sector floricultor?}


Para poder responder este cuestionamiento ya hemos revisado el sector floricultor colombiano, sus fortalezas y debilidades, su posición con respecto a sus competidores. Ahora bien, también hemos entendido los conceptos de cadena de valor y diferenciamos el concepto de cadena de abastecimiento, pasando más adelante a entender las distintas formas de medir el desempeño de ésta.

Para este caso específico, planteamos un modelo integrado por las tres herramientas descritas, es decir, gSCM, SCOR y Balanced Scoredcard, permitiendo:

1. Diagnosticar a partir del gSCM estableciendo los factores externos que afectan a la cadena de abastecimiento en el corto, mediano y largo plazo, señalando los factores externos que impactan a esta cadena, entre ellos, la tecnología, los precios internacionales del dólar y los precios nacionales de insumos y mano de obra, los precios en fletes y las restricciones que en materia ambiental y comercial puedan afectar el mercado. Esto lo observamos en la capa 1 y en la capa 2.

2. Realizar un análisis de la cadena desde el punto de vista del SCOR, lo que implica pensar en cada eslabón de ésta, integrando, sincronizando y optimizando cada uno de los procesos, teniendo en cuenta flujos de bienes, servicios, información, dinero y decisiones. Capa 3. Lo anterior permite implementar las mejores prácticas, dentro de las cuales tenemos:

- Estimular la flexibilidad en la organización, mediante: la disminución de la clasificación de los cargos, la fuerza de trabajo autodirigida, una estructura organizacional plana, y equipos de trabajo multifuncionales.

- Desarrollar la programación de distribución de forma colaborativa con el cliente. Lo anterior, se puede realizar mediante el Acceso Web al estado de la programación de los cultivos y dado un ambiente colaborativo de intercambio de información entre clientes y productores de flores.

- Publicar los resultados de desempeño, utilizando data warehouse, reportes escritos, base de datos en línea y sistemas integrados

- Establecer el nivel de producción, mediante la planeación de la capacidad (en términos de hectáreas cultivadas, flores cortadas, metros cuadrados cosechados, entre otros) que en este caso se mide a partir de la capacidad de cultivo de la tierra. 
ACTIVOS | REVISTA de LA FACULTAD DE CONTADURía Pública

- Vincular el desempeño individual a las metas organizacionales y en los diferentes eslabones de la cadena, pasando por la compra, llegando a la presiembra, siembra, cultivo, cosecha, post cosecha y distribución final.

- Brindar capacitación formal y continua a los empleados, implementando programas para involucrarlos en el establecimiento de planes y en el desarrollo de estrategias organizacionales, en la que ganen tanto la empresa como los empleados. Es importante resaltar que la capacitación de los trabajadores en este caso reviste gran importancia, dado el nivel de escolaridad que en la actualidad ellos tienen

- Reflejar en la programación el estado actual de la planta (nivel de utilización de las instalaciones, áreas de cultivo, áreas de almacenamiento, cuartos fríos, entre otros).

- La programación optimizada del uso de recursos como terrenos, herramientas equipos de propagación, poscosecha y almacenamiento. Esto se puede lograr utilizando las técnicas de optimización.

- Crear manuales de proceso que incluyan los recursos requeridos para producción. Esto es importante dado lo especializado de este sector.

- Mantenimiento integrado de datos que permita asegurar la confiabilidad de los datos de producción, los niveles de inventario de insumos y los requerimientos de demanda.

- Mantener un stock de seguridad estratégico de insumos, para que la obtención del producto no dependa del lead-time del proveedor. Lo anterior es importante con relación a los insumos de producción de las flores.

- Utilización de técnicas de justo a tiempo que permita reducir los tiempos entre los procesos de cosecha y entrega final de las flores.

- Mantener información histórica precisa de lotes cosechados o en proceso de cosecha. Lo anterior puede lograse mediante la recolección electrónica de datos de acciones de los empleados y lotes de producción en compras/proceso, terminados.

- El establecimiento de estándares y medidas alineadas que permitan maximizar el desempeño de la cadena de abastecimiento. Lo anterior, a través del Benchmarking interno/ externo, donde se compare los estándares de la industria, los acuerdos alineados con los clientes y los proveedores, y la visión de los indicadores claves de desempeño. 
- Sistemas de reporte en tiempo real de las medidas de desempeño. Lo anterior permite recolectar información de producción en línea, generar reportes requeridos y hacerle un seguimiento a la producción actual para compararla con la programación y los estándares.

- Utilizar sistemas de información empresariales.

- Manejar procesos de mejoramiento continuo. Que tenga en cuenta tendencias históricas, análisis de causa y efecto, indicadores de desempeño claves. Lo que implica programar revisiones de procesos para encontrar posibles mejoras.

3. Una vez llegamos a este punto, todos los conceptos analizados se enmarcan dentro del modelo de gestión del Balanced Scorecard, en el cual alineamos tanto la estrategia de la cadena de abastecimiento como el resultado de nuestro análisis del sector y sus necesidades.

Veamos gráficamente nuestro planteamiento para el sector de las flores:

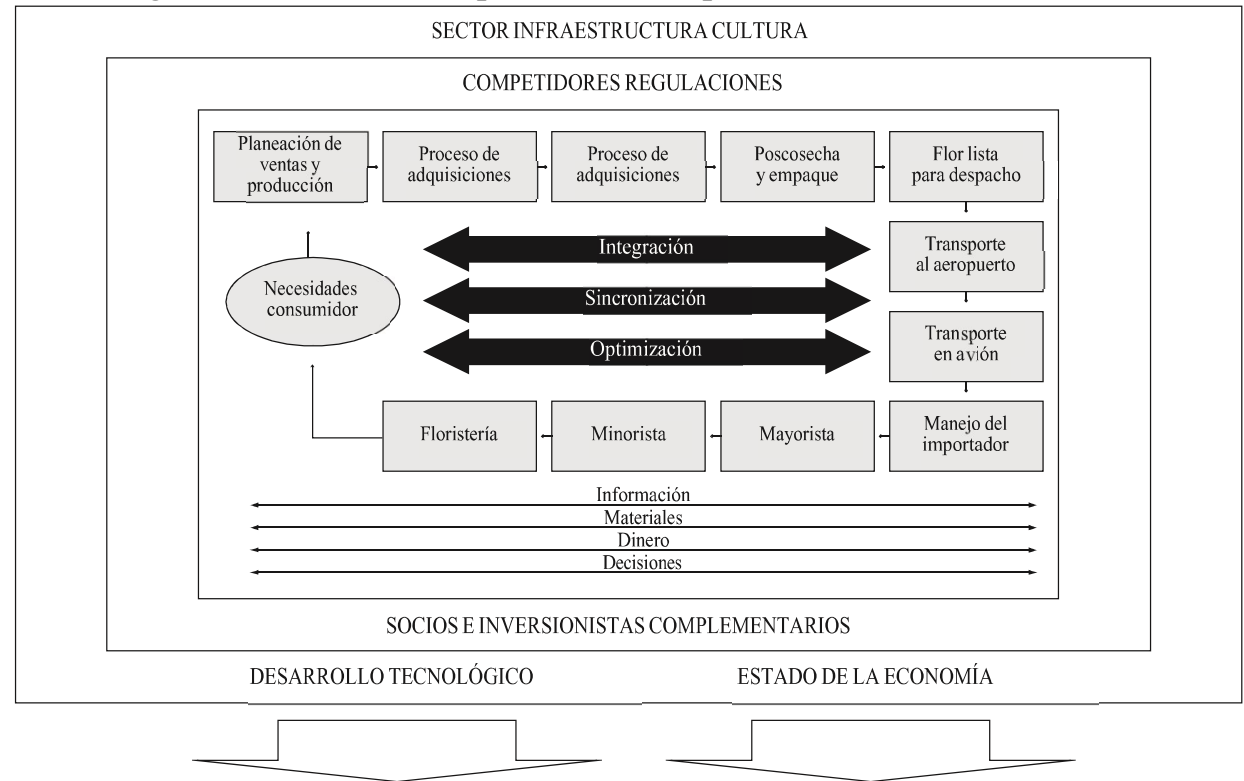

Figura 7. Modelo de definición para el sector floricultor colombiano

Fuente: elaboración propia. 
ACTIVOS | REVista de la Facultad de CONTAduría PÚBlica

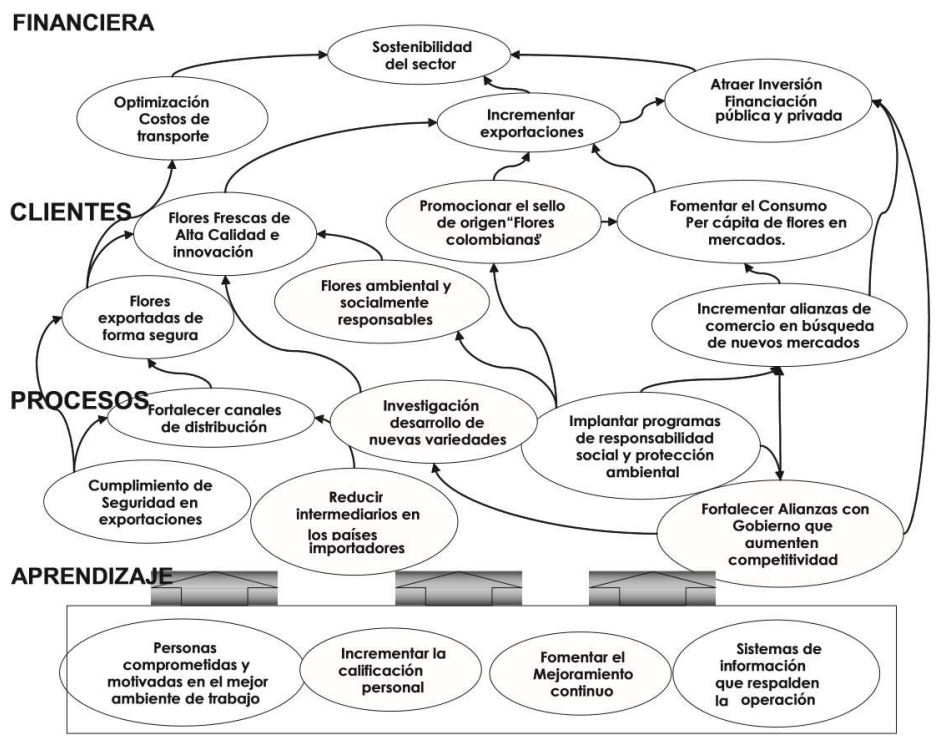

Figura 8. Mapa estratégico para el sector floricultor Fuente: elaboración propia.

Dentro de los objetivos propuestos en el mapa estratégico consideramos importante enfatizar en los siguientes:

- Optimización de costos de transporte

- Promocionar el sello de origen "Flores Colombianas"

- Fortalecer canales de distribución

- Investigación y desarrollo de nuevas variedades

- Reducir intermediarios en los países importadores

- Sistemas de información que respalden la operación

Debido a que son estos objetivos los que permiten que la cadena de abastecimiento del sector floricultor se diferencie y logre mayor competitividad, de acuerdo con nuestro análisis.

Finalmente, concluimos nuestro análisis con el planteamiento de algunos indicadores que permitan medir el cumplimiento de los objetivos estratégicos, y el desempeño en la cadena y sus diferentes eslabones con relación a las estrategias e iniciativas desarrolladas: 
Tabla 2. Indicadores que permiten medir el cumplimiento de los objetivos estratégicos y el

\begin{tabular}{|c|c|c|}
\hline PERSP. & OBJETIVO & INDICADORES \\
\hline \multirow{5}{*}{ 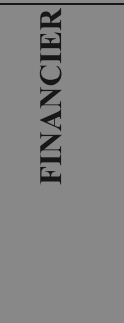 } & Sostenibilidad del sector & $\mathrm{EVA}^{12}$ \\
\hline & Optimización de costos de transporte & Flete por kilo (aéreo y terrestre) \\
\hline & \multirow{2}{*}{ Incrementar exportaciones } & \% Incremento de exportaciones en dólares \\
\hline & & \% Incremento de exportaciones en volumen \\
\hline & $\begin{array}{l}\text { Atraer inversión y financiación } \\
\text { pública y privada }\end{array}$ & $\begin{array}{l}\text { Incremento en créditos concedidos por el } \\
\text { sector financiero }\end{array}$ \\
\hline \multirow{4}{*}{ 穿 } & \multirow{3}{*}{$\begin{array}{l}\text { Flores frescas de alta calidad e } \\
\text { innovación }\end{array}$} & \% Devoluciones por calidad \\
\hline & & \% Satisfacción de clientes \\
\hline & & Incremento de ventas de nuevas variedades \\
\hline & Flores exportadas de forma segura & $\begin{array}{l}\text { \% Empresas de la cadena de abastecimiento } \\
\text { que son BASC }\end{array}$ \\
\hline \multirow[t]{3}{*}{$\sum_{\bar{y}}^{\bar{Z}}$} & $\begin{array}{l}\text { Flores ambiental y socialmente } \\
\text { responsables }\end{array}$ & $\begin{array}{l}\text { Nivel de percepción del cliente sobre las } \\
\text { flores colombianas en cuanto al respeto por } \\
\text { el medio ambiente y los derechos laborales }\end{array}$ \\
\hline & $\begin{array}{l}\text { Promocionar el sello de origen } \\
\text { "Flores Colombianas" }\end{array}$ & $\begin{array}{l}\% \text { Gastos en publicidad en el exterior con } \\
\text { respecto a la venta }\end{array}$ \\
\hline & $\begin{array}{l}\text { Fomentar el consumo per-cápita de } \\
\text { las flores en el exterior }\end{array}$ & Incremento del consumo per-cápita \\
\hline \multirow{5}{*}{ 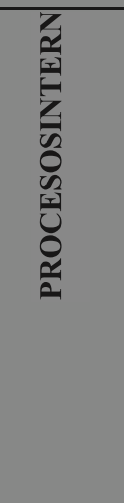 } & $\begin{array}{l}\text { Investigación y desarrollo de nuevas } \\
\text { variedades }\end{array}$ & $\begin{array}{l}\text { \% Nuevas variedades desarrolladas en } \\
\text { Colombia del total de nuevas variedades } \\
\text { manejadas }\end{array}$ \\
\hline & $\begin{array}{l}\text { Incrementar alianzas de comercio en } \\
\text { búsqueda de nuevos mercados }\end{array}$ & $\begin{array}{l}\text { Número de convenios internacionales para } \\
\text { exportación de flores }\end{array}$ \\
\hline & \multirow{3}{*}{$\begin{array}{l}\text { Implantar programas de } \\
\text { responsabilidad social y protección } \\
\text { ambiental }\end{array}$} & \% Empresas certificadas con Florverde \\
\hline & & $\begin{array}{l}\text { \% Empresas que manejan sistemas de } \\
\text { gestión ambiental }\end{array}$ \\
\hline & & $\begin{array}{l}\text { \% Empresas que manejan sistemas de } \\
\text { gestión en salud ocupacional y seguridad } \\
\text { industrial }\end{array}$ \\
\hline
\end{tabular}

${ }^{12}$ EVA Economic Value Added, valor económico agregado representa la riqueza generada para los accionistas y se calcula restándole a la utilidad operacional antes de impuestos, el costo financiero producido por tener activos. 


\begin{tabular}{|c|c|c|}
\hline & $\begin{array}{l}\text { Fortalecer alianzas con gobierno que } \\
\text { aumenten competitividad }\end{array}$ & $\begin{array}{l}\text { Cumplimiento de cronograma establecido en } \\
\text { la agenda de competitividad con el sector } \\
\text { público }\end{array}$ \\
\hline \multirow{4}{*}{ 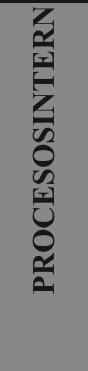 } & \multirow{3}{*}{ Fortalecer canales de distribución } & $\begin{array}{l}\% \text { Pedidos cumplidos en o antes de la fecha } \\
\text { requerida por el cliente }\end{array}$ \\
\hline & & $\begin{array}{l}\% \text { Pedidos cumplidos en o antes de la fecha } \\
\text { original programada o comprometida }\end{array}$ \\
\hline & & \% Pedidos perfectos \\
\hline & $\begin{array}{l}\text { Reducir intermediarios en los países } \\
\text { importadores }\end{array}$ & $\begin{array}{l}\text { Número de intermediarios promedio desde la } \\
\text { llegada de la flor en puerto hasta su venta al } \\
\text { consumidor final }\end{array}$ \\
\hline \multirow{5}{*}{ 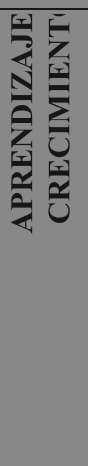 } & \multirow[b]{2}{*}{$\begin{array}{l}\text { Personas comprometidas y motivadas } \\
\text { en el mejor ambiente de trabajo }\end{array}$} & Rotación del personal \\
\hline & & $\begin{array}{l}\text { \% Personas vinculadas a programas de } \\
\text { bienestar del sector }\end{array}$ \\
\hline & \multirow[t]{2}{*}{ Incrementar calificación del personal } & $\begin{array}{l}\text { \% Personas en el sector favorecidos por algún } \\
\text { convenio educativo con el SENA o las } \\
\text { universidades }\end{array}$ \\
\hline & & \% Inversión en capacitación sobre ventas \\
\hline & Fomentar el mejoramiento continuo & $\begin{array}{l}\text { \% Incentivos otorgados al personal por ideas } \\
\text { que mejoren calidad o eficiencia de los } \\
\text { procesos }\end{array}$ \\
\hline \multirow{3}{*}{ 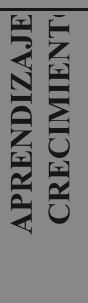 } & \multirow{3}{*}{$\begin{array}{l}\text { Sistemas de información que } \\
\text { respalden la operación }\end{array}$} & $\begin{array}{l}\text { \% Empresas que cuentan con sistemas de } \\
\text { información eficientes }\end{array}$ \\
\hline & & $\begin{array}{l}\text { Cumplimiento de cronograma para el } \\
\text { establecimiento en el sector de un nuevo } \\
\text { sistema de información }\end{array}$ \\
\hline & & $\begin{array}{l}\text { \% Empresas que cuentan con sistemas de } \\
\text { información eficientes }\end{array}$ \\
\hline
\end{tabular}

Fuente: elaboración propia.

\section{Conclusiones}

Con la realización de esta investigación podemos concluir que el sector floricultor colombiano, es un sector estratégico para la economía de nuestro país, considerando que estos productos tienen posibilidades de expansión en los mercados mundiales.

Sin embargo, vale la pena resaltar que para mantener los mercados actuales y conquistar nuevos mercados, se deben superar los problemas actuales que limitan 
la capacidad de las organizaciones de flores colombianas para ser competitivas, dadas las condiciones de internacionalización económica. Entre estos problemas encontramos, como se mencionó en el diagnóstico del sector, debilidades tecnológicas, falta de integración a lo largo de la cadena de abastecimiento, problemas en la distribución y conservación de las flores, problemas en los sistemas de información, y la inexistencia de herramientas de medición del desempeño, entre otros.

Para la superación de estas debilidades y en procura de afrontar con éxito las condiciones actuales de competencia, consideramos que se requiere del conocimiento y de la gestión de la cadena de abastecimiento, en la cual se planee, compre, genere y entregue, a partir de la implementación de principios de colaboración, integración, sincronización y optimización de los recursos involucrados a lo largo de la cadena.

El desarrollo adecuado de este proceso exige, el uso de herramientas, que permitan medir el desempeño a lo largo de la cadena de abastecimiento. Planteamos entonces, varios modelos que podemos integrar, para que apoyen la gestión en este sector, el modelo GSCM, el modelo SCOR y el Balanced Scoredcard. Se propone integrar estas herramientas y buscar alinear la estrategia de los procesos de la cadena, con sus diferentes actores, recursos involucrados y con la implementación de mejores prácticas aplicadas al sector.

También resaltamos como conclusiones importantes para la competitividad del sector los siguientes aspectos, que planteamos como objetivos del mapa estratégico:

- Impulsar en el exterior el sello de origen "Flores Colombianas" para fortalecer la presencia en estos mercados, generando una imagen respaldada en la coordinación de la cadena e integración de sus actores, buscando con esto cumplir la promesa de valor a los clientes.

- Fortalecer los canales de distribución utilizando esquemas de colaboración y sistemas de información integrados vía Intercambio Electrónico de Datos (EDI).

- Reducir intermediarios en los países importadores, con esto se busca llegar directamente al consumidor final, para asegurar su satisfacción directa y conocimiento de sus preferencias y necesidades. 
ACTIVOS | REVISTA de LA FACULTAD DE CONTADURía Pública

- Sistemas de información que respalden la operación, permitiendo así la comunicación directa con el cliente y con cada uno de los que participan en la cadena de abastecimiento, buscando de esta manera la satisfacción y la generación de valor para todos.

- Optimizar costos de transporte, debido a que uno de los mayores generadores de costo son los fletes aéreos y terrestres, dados los requerimientos en el transporte de las flores (transporte en frío) para conservar su calidad.

- Investigación y desarrollo de nuevas variedades, consideramos este objetivo de importancia, ya que, éste debe orientar a la organización en la conquista de nuevos mercados, alcanzando el posicionamiento esperado, al ofrecer productos que no sea tan fácil ubicar en los mercado actuales.

Finalmente, esperamos con este trabajo contribuir de una $\mathrm{u}$ otra manera, al desarrollo de nuestro país, ya que si este sector se fortalece, tendrá efecto directo en nuestra economía. De otra parte, para el sector al que fue dirigido el presente trabajo, debe convertirse en una herramienta que le permita gestionar el negocio con un nuevo enfoque, cuyas directrices están descritas a lo largo del trabajo, buscamos además que nuestra propuesta les permita lograr los objetivos que proponemos a partir del mapa estratégico del Balanced Scoredcard.

\section{Referencias}

¿Qué es la cadena de abastecimiento? (2003). Revista Administración de la cadena de suministros. La República.

Acero, M. (2003). Las cadenas de suministro del sector agropecuario y el TLC. Revista Administración de la cadena de suministros. La República.

Balanced Scorecard y las cadenas de suministros (2004). Revista Administración de la cadena de suministros. La República.

Ballou, R. (2004). Business logistics. Supply chain management: planning, organizing, and controlling the supply chain. New Jersey: Prentice Hall.

Características críticas de las cadenas agroindustriales (2005). Revista Administración de la cadena de suministros. La República. 
Carranza, O., \& otros. (2004). Logística: mejores prácticas en Latinoamérica. México: Editorial Thomson.

Centro Documental Asociación Colombiana de Floricultores ASOCOLFLORES.

Chopra, S. \& Meindl, P. (2004). Supply chain management: Strategy, Planning, and Operation. New Jersey: Prentice Hall.

Competitividad en integración de supply Chaín y logística en Colombia (2003). Revista Administración de la cadena de suministros. La República.

Esquemas de planeación en la cadena de suministros (2004). Revista Administración de la cadena de suministros. La República.

Estrategias en la cadena de valor (2003). Revista Administración de la cadena de suministros. La República.

Hakanson, B. (2003). Manejo de la cadena de abastecimiento: donde compiten los negocios hoy. Revista Administración de la cadena de suministros. $L a$ República.

Importancia de la gestión logística y de cadenas de suministro de la empresa colombiana frente al TLC (2005). Revista Administración de la cadena de suministros. La República.

Infraestructura de cadena de suministro para el TLC (2004). Revista Administración de la cadena de suministros. La República.

Institute of Management Accountants. IMA. (1992). Certified Management Accountant Program. Cost management for logistics. New York.

Kaplan, R. \& Cooper, R. (2005). Coste y efecto: cómo usar el ABC, el ABM y el $A B B$ para mejorar la gestión, los procesos y la rentabilidad.

Las cadenas de suministro del sector agropecuario (2005). Revista Administración de la cadena de suministros. La República. 
ACTIVOS | REVISTA DE LA FACULTAD DE CONTADURÍA PÚBLICA

Mallo, C. Kaplan, R, Meljem, S \& Jiménez, C. (2000). Contabilidad de costos y estrategia de gestión. Madrid: Prentice may Iberia.

Mariño, F. (2003). Óptimos globales y cadena de suministro. Revista Administración de la cadena de suministros. La República.

Martínez L, \& González A. (2005). Propuesta de mejoramiento de los procesos de producción y distribución de la cadena de abastecimiento de Restcafé OMA S.A. en las barras de café a través de la aplicación del modelo SCOR. (Tesis de grado). Pontificia Universidad Javeriana, Facultad de Ingeniería Industrial.

Metodología colombiana para implementar SCM (2006). Revista Administración de la cadena de suministros. La República.

Ministerio de Comercio Exterior. (s/f). Dirección de competitividad. Agenda de competitividad: Estudio sobre el sector flores. www.mincomex.gov.co.

Modelo analítico para el análisis de una cadena (2003). Revista Administración de la cadena de suministros. La República.

Modelo de sistemas de información empresarial para SMC (2003). Revista Administración de la cadena de suministros. La República.

Pasos para diseñar una cadena de suministros en el sector agroindustrial (2005). Revista Administración de la cadena de suministros. La República.

Qué tanto necesitamos administrar una cadena (2003). Revista Administración de la cadena de suministros. La República.

Relaciones de colaboración en la cadena de suministro (2006). Revista Administración de la cadena de suministros. La República.

SCOR un modelo para ser más competitivos (2006). Revista Administración de la cadena de suministros. La República.

Sierra, P. (2003). Floricultura de exportación en América Latina: hipótesis y retos. Editorial Cactus. 
Suppli, C. (2005). Busca asociados para aplicar modelo de referencia SCOR. Revista Administración de la cadena de suministros. La República.

Tenjo, F; Montes, E. \& Martínez, J. (2006). Comportamiento Reciente (20002005) del sector floricultor colombiano.

Young, R. (2005). Vulnerabilidades de la cadena de suministros: consideraciones para el caso de América Latina. Revista Latinoamericana de Administración. 